\title{
Hypoceruloplasminemia: an unusual biochemical finding in a girl with Hashimoto's thyroiditis and severe hypothyroidism
}

\author{
Mariella Valenzise, ${ }^{1}$ Federica Porcaro, ${ }^{2}$ Giuseppina Zirilli, ${ }^{1}$ Filippo De Luca, ${ }^{1}$ Maurizio Cinquegrani, ${ }^{3}$ \\ Tommaso Aversa 1 \\ ${ }^{1}$ Department of Human Pathology of Adulthood and Childhood, University of Messina, Messina; ${ }^{2}$ Respiratory Unit, \\ Academic Department of Pediatrics, Bambino Gesù Children's Hospital, Rome; ${ }^{3}$ Department of Clinical and \\ Experimental Medicine, University of Messina, Messina, Italy
}

\begin{abstract}
Clinical picture of Hashimoto's thyroiditis (HT) may significantly vary in pediatric age, ranging from euthyroidism to subclinical hypothyroidism or hyperthyroidism; only rarely HT presentation may be characterized by a severe hypothyroidism also in pediatric age. Here we describe a 3-year-old Caucasian girl who was admitted to our Clinic due to pericardial effusion, muscle weakness and weight gain. At clinical examination, she presented with bradycardia, pale and round face, pseudohypertrophy of calf muscles and no pitting edema of the limbs. Routine blood investigations showed high serum aspartate and alanine aminotransferase levels, low serum ceruloplasmin without clinical signs of Wilson's disease, dyslipidemia. Thyroid function tests revealed a picture of severe hypothyroidism associated with HT. After the replacement
\end{abstract}

Correspondence: Valenzise Mariella, Department of Human Pathology of Adulthood and Childhood, University of Messina, Via Consolare Valeria, 98125 Messina, Italy.

Tel.: +39.090.2213154.

E-mail: mvalenzise@unime.it

Key words: Hashimoto's thyroiditis, Thyroid function evolution; Thyroid status prognosis; Hypoceruloplasminemia.

Contributions: MV and FDL wrote the paper; TA and GZ organized the material and prepared its distribution in the different sections; FP prepared the graphics and collected references; $\mathrm{MC}$ performed the ultrasound evaluation.

Conflict of interest: the authors declare no potential conflict of interest.

Funding: none.

Ethical statement: the informed consent has been obtained from the parents of our patient.

Received for publication: 9 November 2017

Accepted for publication: 8 May 2018.

This work is licensed under a Creative Commons Attribution

NonCommercial 4.0 License (CC BY-NC 4.0).

(C) Copyright M. Valenzise et al., 2018

Licensee PAGEPress, Italy

La Pediatria Medica e Chirurgica 2018; 40:179

doi:10.4081/pmc.2018.179 treatment with L-T4, thyroid-stimulating hormone serum levels gradually decreased, with concomitant resolution of pericardial effusion and normalization of ceruloplasmin levels.

\section{Introduction}

Hashimoto's thyroiditis (HT) is the most common thyroid disease in childhood and the most frequent cause of thyroid enlargement and acquired hypothyroidism in children and adolescents from iodine replete areas. Both thyroid function tests and clinical manifestations at HT presentation may significantly vary in the different pediatric cohorts. ${ }^{1-6}$

In children euthyroidism is the most frequent biochemical pattern at diagnosis, ${ }^{7}$ followed by subclinical hypothyroidism, overt hypothyroidism and, more rarely, either subclinical or overt hyperthyroidism. ${ }^{1-5,8,9}$

From a clinical view the most common presenting manifestation is goiter, but most children may also be asymptomatic at the time of diagnosis. 6 Other less common manifestations are those originating from compression of the cervical structures that are anatomically contiguous to thyroid gland and include cough, hoarseness, dysphagia, dysphonia, and dyspnea. ${ }^{6}$

In the children presenting with a biochemical picture of overt hypothyroidism it is possible to observe, at diagnosis, clinical symptoms of thyroid function impairment: bradycardia, constipation, changes of skin and appendages, hypochromic and microcytic anemia and neuropsychiatric symptoms, such as attention loss and impaired scholastic performances. ${ }^{6}$ In a $3.5 \%$ of cases HT may present with a transient hyperthyroid picture and such a presentation pattern is known as hashitoxicosis, which is the second commonest cause of hyperthyroidism in childhood. ${ }^{10}$ In these cases differential diagnosis between Graves' disease and HT may be sometimes very difficult, at least on the basis of clinical examination. $8,10,11$

However, it is well known that HT presentation in childhood may be very heterogeneous and many other clinical and biochemical abnormalities have been reported to be detectable in a child presenting HT. $12-17$

We have just recently observed a girl, who exhibited, at the time of HT diagnosis, a biochemical finding (hypoceruloplasminemia) that had never been reported to be possibly associated with this disease. This atypical finding gave us the opportunity of analyzing the physiological role of thyroid hormones in the mechanism of serum ceruloplasmin level regulation. 


\section{Case Report}

A 3-year-old girl was admitted to our Clinic due to a clinical picture characterized by recurrent pericarditis, fatigue and muscle weakness. Her family history was remarkable for both autoimmune thyroid diseases and hypercholesterolemia. Her recent personal history was significant for weight gain (about $2 \mathrm{Kg}$ ), face swelling and chronic constipation during the last two months.

At clinical examination she exhibited: pale, yellowish and round face, hypertrophy of calf muscles and no pitting edema of the limb. Abdomen was protruding, meteoric and soft at palpation. Neurological examination was negative and Gower's sign was absent. Thyroid size was normal and no nodules and/or regional lymphoadenopathies were detected at physical examination.

Echocardiogram, performed at the first day of hospitalization, showed thickening and minimal dissection of pericardium. Laboratory investigations showed increased serum concentrations of liver enzyme specially aspartate aminotransferases, accompanied by elevated creatine kinase (CK) levels. Total and LDL cholesterol were high, whereas triglyceride levels were normal (Table 1).

In order to identify the possible causes of hypertransaminasemia, we performed the following laboratory investigations: toxoplasma and viral serology (Epstein Barr, Cytomegalovirus, Herpes, Hepatitis A, Hepatitis B and Hepatitis C), serology for celiac disease, analysis of the specific autoimmune panel (antinuclear, antismooth muscle, liver-kidney microsomal and extractable nuclear antibodies). All these investigations were negative.

Laboratory investigations showed also low ceruloplasmin levels (Table 1). In order to exclude Wilson disease, our patient underwent to 24-hour urinary copper and serum-free copper detection resulting normal; the abdominal ultrasound was also negative and the ophtalmological evaluation excluded the presence of the Keiser Fleischer ring.

Thyroid function tests showed very high thyroid-stimulating hormone (TSH) levels and low FT4 values (Table 1).

Anti-thyroid peroxidase autoantibody (TPOAb) titers were very high whilst antithyroglobulin autoantibody titers were within reference range (Table 1).

Thyroid ultrasound evaluation showed a third grade sonographic pattern according to Sostre's classification. ${ }^{18}$

On the basis of clinical picture and diagnostic assessments we established a diagnosis of HT-related severe hypothyroidism with pericardial effusion, myopathy, hypercholesterolemia and hypoceruloplasminemia. Therefore, we started a replacement treat- ment with L-T4. Laboratory investigations were repeated three weeks after therapy onset and evidenced a normalization of all the pathological biochemical findings (Table 1). Also the clinical and echocardiographic abnormalities regressed under L-T4 therapy.

On the light of the overall improvement which was recorded under L-T4 replacement treatment, the initial diagnosis of HTrelated hypothyroidism was finally confirmed.

\section{Discussion}

Whereas the presentation modes of HT in childhood are mainly conditioned by patients' age at the time of diagnosis, ${ }^{7}$ its evolution over time is mainly affected by TSH serum levels at presentation 19,20 and additional risk factors, such as the coexistence with either celiac disease or very elevated TPOAb serum levels ${ }^{19}$ or Turner syndrome. ${ }^{21-23}$

Although it is largely accepted that HT in the majority of children and adolescents presents with a mild biochemical and clinical picture, ${ }^{1-9}$ nevertheless it is also known that, in a minority of cases, biochemical and clinical presentation may be very severe, ${ }^{12-17}$ as in the present report.

Our patient referred to our Clinic due to a clinical picture characterized by pericarditis, muscle weakness and fatigue. The pathophysiological mechanism of pericardial effusion in hypothyroidism lies on both an increased capillary permeability and an impaired lymphatic drainage, with subsequent leakage of proteins into the interstitial space. However, this manifestation is known to occur only in the cases with a severe impairment of thyroid function. ${ }^{14-16,24}$

By contrast, myopathy is a common presenting manifestation in children with overt hypothyroidism and may be clinically asymptomatic and characterized only by mild to moderate CK elevation. ${ }^{17}$

The most unusual aspect of the present case report was the finding of a very low serum concentration of ceruloplasmin at HT diagnosis, a biochemical abnormality that had never been reported to date in a child with hypothyroidism, at least to the best of our knowledge. Such an atypical finding is not easy to be explained from a pathophysiological view. It has to be underlined, however, that thyroid hormones have been recently postulated to play some role in the mechanism of copper serum level regulation, by enhancing both biosynthesis and export of hepatic ceruloplasmin protein into the serum. ${ }^{25,26}$ In fact, ceruloplasmin serum levels have been reported to be abnormally low also in a series of patients with peripheral resistance to thyroid hormones. ${ }^{26}$

Because of low levels of ceruloplasmin are not always indica-

Table 1. Laboratory investigations at diagnosis and under therapy.

\begin{tabular}{lccc} 
Analytes & Diagnosis & Under therapy & Normal range \\
Creatine kinase (U/L) & 418 & 125 & $0-200$ \\
Alanine aminotrasferase (U/L) & 68 & 26 & $0-42$ \\
\hline Aspartate aminotrasferase (U/L) & 107 & 16 & $0-50$ \\
Total cholesterol (mg/dL) & 384 & 202 & $130-220$ \\
\hline Low density lipoprotein cholesterol (mg/dL) & 284 & 126 & $70-160$ \\
Serum cerulopasmin (mg/dL) & 12 & 36.7 & $20-60$ \\
\hline Thyroid stimulating hormone (mIU/mL) & 338.000 & 7.69 & $0.27-4.2$ \\
Free thyroxine (pmol/L) & 3.2 & 21,14 & $12-22$ \\
\hline Anti-thyroid peroxidase antibody (UI/mL) & 2373.00 & 1710 & $0-34$ \\
Anti-thyroglobulin antibody (Ul/mL) & 15.60 & & $0-115$ \\
\hline
\end{tabular}


tive of a copper storage disorder, a correct differential diagnosis with disorders sharing this laboratory findings - such as congenital disorder of glycosylation - may be challenging. ${ }^{27}$

On the basis of that report and the present case history, we infer that hypoceruloplasminemia might be proposed as an additional biomarker for both hypothyroidism and resistance to thyroid hormones. This inference seems to be reinforced by the normalization of serum ceruloplasmin concentrations that accompanied the normalization of thyroid function tests after the onset of L-T4 replacement therapy.

\section{Conclusions}

On the light of this case history we underlie: i) the importance of biochemical screening for hypothyroidism in children presenting with an unexplained pericardial effusion; ii) the importance of maintaining a high level of suspicion for long-lasting hypothyroidism in children with nonspecific complaints of fatigue, myalgia and generalized weakness; iii) the crucial role of thyroid hormones in the regulation of ceruloplasmin levels; therefore the pediatricians should suspect a hypothyroid condition in young patients with hypoceruloplasminemia and no other signs of Wilson's disease.

\section{References}

1. Gopalakrishnan S, Chugh PK, Chhillar M, et al. Goitrous autoimmune thyroiditis in a pediatric population: a longitudinal study. Pediatrics 2008;122:e670-4.

2. Demirbilek H, Kandemir N, Gonc EN, et al. Assessment of thyroid function during the long course of Hashimoto's thyroiditis in children and adolescents. Clin Endocrinol (Oxf) 2009;71:451-4.

3. Zak T, Noczyńska A, Wasikowa R, et al. Chronic autoimmune thyroid disease in children and adolescents in the years 19992004 in Lower Silesia, Poland. Hormones 2005;4:45-8.

4. Ozen S, Berk Ö, Şimşek DG, Darcan S. Clinical course of Hashimoto's thyroiditis and effects of levothyroxine therapy on the clinical course of the disease in children and adolescents. J Clin Res Pediatr Endocrinol 2011;3:192-7.

5. Skarpa V, Kousta E, Tertipi A, et al. Epidemiological characteristics of children with autoimmune thyroid disease. Hormones (Athens) 2011;10:207-14.

6. De Luca F, Aversa T, Salzano G, et al. Autoimmune thyroiditis. In: Bona G, De Luca F, Monzani A, eds. Thyroid diseases in childhood: recent advances from basis science to clinical practice. Switzerland: Springer International Publishing; 2015. pp 181-194.

7. Wasniewska M, Corrias A, Salerno M, et al. Thyroid function patterns at Hashimoto's thyroiditis presentation in childhood and adolescence are mainly conditioned by patients' age. Horm Res Paediatr 2012;78:232-6.

8. Wasniewska M, Corrias A, Salerno M, et al. Outcomes of children with hashitoxicosis. Horm Res Paediatr 2012;77:36-40.

9. Aversa T, Valenzise M, Corrias A, et al. Subclinical hyperthyroidism when presenting as initial manifestation of juvenile
Hashimoto's thyroiditis: first report on its natural history. J Endocrinol Invest 2014;37:303-8.

10. Williamson S, Greene SA. Incidence of thyrotoxicosis in childhood: a national population based study in the UK and Ireland. Clin Endocrinol (Oxf) 2010;72:358-63.

11. Nabhan ZM, Kreher NC, Eugster EA. Hashitoxicosis in children: clinical features and natural history. J Pediatr 2005;146:533-6.

12. Kisakol G, Tunc R, Kaya A. Rhabdomyolysis in a patient with hypothyroidism. Endocr J 2003;50:221-3.

13. Kuo HT, Jeng CY. Overt hypothyroidism with rhabdomyolysis and myopathy: a case report. Chin Med J 2010;123:633-7.

14. Martinez-Soto T, Deal C, Stephure D, et al. Pericardial effusion in severe hypothyroidism in children. J Pediatr Endocrinol Metab 2010;23:1165-8.

15. Delgado Hurtado JJ, Guevara W, Ramos E, et al. Hypothyroidism in a five-year-old boy with rhabdomyolysis and recent history of cardiac tamponade: a case report. J Med Case Rep 2011;5:515.

16. Dursun F, Güven A, Ayhan YI. Kocher-Debré-Sémélaigne syndrome with pericardial effusion. J Pediatr Endocrinol Metab 2011;24:1099-101.

17. Serranti D, Indolfi G, Bartolini E, et al. Raised serum aminotransferase levels and muscle pseudohypertrophy caused by hypothyroidism. J Pediatr Gastroenterol Nutr 2013;56:e48-9.

18. Sostre S, Reyes MM. Sonographic diagnosis and grading of Hashimoto's thyroiditis. J Endocrinol Invest 1991;14:115-21.

19. Radetti G, Maselli M, Buzi F, et al. The natural history of the normal/mild elevated TSH serum levels in children and adolescents with Hashimoto's thyroiditis and isolated hyperthyrotropinaemia: a 3-year follow-up. Clin Endocrinol (Oxf) 2012;76:394-8.

20. Aversa T, Corrias A, Salerno M, et al. Five-year prospective evaluation of thyroid function test evolution in children with Hashimoto's thyroiditis presenting with either euthyroidism or subclinical hypothyroidism. Thyroid 2016;26:1450-6.

21. Aversa T, Lombardo F, Valenzise M, et al. Peculiarities of autoimmune thyroid diseases in children with Turner or Down syndrome: an overview. Ital J Pediatr 2015;41:39.

22. Wasniewska M, Aversa T, Salerno M, et al. Five year prospective evaluation of thyroid function in girls with subclinical mild hypothyroidism of different etiology. Eur J Endocrinol 2015;173:801-8.

23. Wasniewska M, Salerno M, Corrias A, et al. The evolution of thyroid function after presenting with Hashimoto thyroiditis is different between initially euthyroid girls with and those without Turner syndrome. Horm Res Paediatr 2016;86:403-9.

24. Imazio M, Mayosi BM, Brucato A, et al. Triage and management of pericardial effusion. J Cardiovasc Med (Hagerstown) 2010;11:928-35.

25. Fitch CA, Song Y, Levenson CW. Developmental regulation of hepatic ceruloplasmin mRNA and serum activity by exogenous thyroxine and dexamethasone. Proc Soc Exp Biol Med 1999;221:27-31.

26. Mittag J, Behrends T, Nordstrom K, et al. Serum copper as a novel biomarker for resistance to thyroid hormone. Biochem J 2012;443:103-9.

27. Nicastro E, Ranucci G, Vajro P, et al. Re-evaluation of the diagnostic criteria for Wilson disease in children with mild liver disease. Hepatology 2010;52:1948-56. 